\title{
A INCLUSÃO NO CONTEXTO DIGITAL POR MEIO DO USO DE LEITORES DE TELA: CRIAÇÃO E IMPLEMENTAÇÃ DE NOVOS RECURSOS AO LEITOR DE TELA NVDA
}

\author{
Davi Braga da Cruz - davibc19@ hotmail.com - Universidade Federal de Itajubá (UNIFEI) \\ Profa. Dra. Paloma Alinne A.Rodrigues - palomaraap@unifei.edu.br - Universidade Federal \\ de Itajubá (UNIFEI)
}

\begin{abstract}
Resumo: A literatura revela que foram ampliadas as discussões sobre a necessidade de investir esforços para garantir o pleno acesso das pessoas com deficiência a todas as esferas da sociedade. Nesse ínterim é preciso pensar em recursos que potencializem a inclusão de pessoas com Deficiência Visual (DV) ao contexto digital. Nesse processo, a pessoa com DV passa a ter a oportunidade de desenvolver diferentes atividades como a possibilidade de realizar um curso na modalidade de Educação a Distância (EaD). Todavia, para que isso aconteça à pessoa com DV, que está iniciando suas atividades, necessita compreender as funcionalidades dos leitores de tela. Desse modo, foi desenvolvido, no âmbito do grupo de pesquisa Núcleo de Estudos em Formação Docente, Tecnologias e Inclusão (NEFTI) da Universidade Federal de Itajubá (UNIFEI) um projeto de Iniciação Científica ${ }^{1}$ que visava criar e implementar novos recursos a um menu auxiliar desenvolvido, em uma etapa inicial da pesquisa no ano de 2015, para o leitor de tela NonVisual Desktop Access (NVDA). Sendo assim, o objetivo desse trabalho e mostrar os resultados alcançados nessa fase da pesquisa e disponibilizar para a comunidade o endereço de acesso ao software desenvolvido.
\end{abstract}

Palavras-chave: deficiência visual, tecnologia assistiva, inclusão, leitor de tela

Abstract: The literature reveals that discussions have been broadened on the need to invest in efforts to ensure full access for people with disabilities to all spheres of society. In the meantime it is necessary to think of resources that enhance the inclusion of people with Visual Impairment (DV) to the digital context. In this process, the person with DV will have the opportunity to develop different activities such as the possibility of taking a course in the modality of Distance Education (EAD). However, for this to happen to the person with DV, who is starting their activities, he needs to understand the functionalities of the screen readers. Thus, a project of Scientific Initiation was developed within the framework of the Research Group on Teaching Training, Technologies and Inclusion (NEFTI) of the Federal University of Itajubá (UNIFEI), aimed at creating and implementing new resources for a developed auxiliary menu, At an early stage of research in the year 2015, for the NonVisual Desktop Access (NVDA) screen reader. Thus, the objective of this work is to show the results achieved in this phase of the research and to provide the community with the access address for the software developed.

Keywords: visual impairment, assistive technology, inclusion, screen reader

\footnotetext{
${ }^{1}$ Os autores agradecem o apoio da Fundação de Amparo à Pesquisa do Estado de Minas Gerais (FAPEMIG)
} 


\section{INTRODUÇÃO}

A Constituição Federal de 1988 assegura no artigo $1^{\circ}$ e $3^{\circ}$ que qualquer individua, independente, da raça, sexo, cor e idade tem direito a cidadania. No artigo $3^{\circ}$ inciso IV um dos objetivos da República Federativa do Brasil, configura-se em promover o bem estar de todos sem preconceitos ou formas de discriminação. Já o artigo 205 assegura que a Educação é um direito de todos e, no artigo 206, garante-se que o ensino deve ser ministrado em igualdade de condições, para que assim, o aluno tenha subsídios para permanecer no ambiente escolar. Todavia, foi apenas em 1994, com a Declaração de Salamanca sobre Princípios, Política e Práticas na Área das Necessidades Educativas Especiais é que se reafirma "o direito à educação de todos os indivíduos [...] reconhecendo a necessidade e urgência de garantir a educação para as crianças, jovem e adulta com necessidades educativas especiais [...]" (Salamanca, 1994). Ao longo dos anos, novas leis que contemplavam a Inclusão foram aprovadas $^{2}$, tanto em âmbito nacional quanto mundial, fazendo com que os conceitos de inclusão e acessibilidade fossem cada vez mais difundidos.

Nesse ínterim, cabe destacar o desenvolvimento de recursos adaptados para potencializar a participação das pessoas com deficiência ao contexto social, digital e educacional. Tais recursos são definidos como Tecnologia Assistiva (TA) e compreendidos como "[...] qualquer item, equipamento ou parte dele, produto ou sistema fabricado em série ou sob medida, utilizado para aumentar, manter ou melhorar as capacidades funcionais das pessoas com deficiência" (Galvão, 2005, p. 207-235).

Entre os diferentes tipos de recursos de TA, neste trabalho, destaca-se os Leitores de Tela, compreendido como software que conta com um sintetizador de voz, encarregado de transformar informações textuais em verbais. As pessoas com DV podem contar, atualmente, com uma diversidade de leitores de telas. No entanto, em alguns casos é necessário investir recursos financeiros para adquiri-los como, por exemplo, o JAWS criado por uma empresa americana. No contexto brasileiro têm-se o leitor de tela Virtual Vision desenvolvido em 1977 e o DOSVOX criado em 1994 pela Universidade Federal do Rio de Janeiro (UFRJ). Este último tem sido indicado para crianças e jovens que estão iniciando suas atividades no mundo digital (Melo, 2010).

Além disso, tem-se o leitor de tela NonVisual Desktop Access (NVDA), foco deste trabalho, que é um software open-source, gratuito, portátil e constantemente atualizado "por uma comunidade internacional motivada no aprimoramento das funcionalidades de softwares desta categoria" (Brasil, 2009, p. 97-98). Cabe destacar que, em uma investigação realizada por Oliveira Júnior (2013) na UFRJ o pesquisador verificou que as pessoas com DV desempenhavam de uma forma mais eficiente às atividades quando utilizavam o leitor de tela NVDA.

Desse modo, o desenvolvimento e o aprimoramento de recursos de TA, como os leitores de tela, permitem as pessoas com DV desenvolver diferentes ações como, por exemplo, acessar a internet, navegar nas redes sociais, ter acesso a notícias e realizar cursos que são ofertados na modalidade de Educação a Distância (EaD), em especial no contexto do Ensino Superior. Devido a isso, no âmbito do grupo de pesquisa Núcleo de Estudos em Formação Docente, Tecnologias e Inclusão (NEFTI) da Universidade Federal de Itajubá (UNIFEI) foi desenvolvido um projeto de Iniciação Científica que teve como intuito criar e implementar ao leitor de tela NVDA um menu auxiliar para otimizar o seu uso por pessoas com DV que iniciavam suas atividades no contexto digital (Cruz e Rodrigues, 2015). Na primeira fase dessa pesquisa, Cruz e Rodrigues (2015) verificaram que a voz sintetizada do

\footnotetext{
2 Lei No 9394 de 1996. Disponível em: http://portal.mec.gov.br/seesp/arquivos/pdf/lei9394_ldbn2.pdf. Acesso em: $24 / 04 / 2017$

Decreto No 3.298 de 1999. Disponível em:http://www.planalto.gov.br/ccivil_03/decreto/D3298.htm. Acesso em: 24/04/2017.
}

V. $15 \mathrm{~N}^{\mathrm{o}} 1$, julho, 2017 
NVDA propiciava ao usuário uma confusão entre as palavras, assim como os usuários sentiam dificuldade para compreender a pronuncia do sintetizador de voz. Diante disso, nesse período foi desenvolvido um menu auxiliar que propiciava ao usuário modificar a voz disponível no leitor de tela e, além disso, o ensinava a navegar no computador e a acessar a internet. Nesse processo, também, foi incluído dentro do menu um item denominado de "recomendações". Tal item dispõe de orientações quanto ao uso dos recursos de acessibilidade do Windows e, também, quanto ao uso das teclas de atalho. Durante o ano de 2016, deu-se continuidade à investigação e, devido a isso, os autores identificaram outras dificuldades que os usuários DV iniciantes possuíam ao utilizar o leitor de tela NVDA. Entre elas, destaca-se o acesso a um servidor de e-mail e a utilização de softwares de desenvolvimento de trabalhos como o pacote Office, sendo eles o Microsoft Word, Microsoft Excel e o Microsoft Powerpoint. Portanto, o objetivo deste artigo é mostrar os resultados relativos a segunda etapa dessa pesquisa que consistiu na criação e implementação de novos recursos ao menu auxiliar para potencializar o uso do leitor de tela NVDA.

\section{METODOLOGIA}

Nessa etapa da pesquisa optou-se por inicialmente realizar um estudo sobre as principais teclas de atalho e recursos de acessibilidade que o Mozilla Thunderbird e os programas do Pacote Office ofereciam aos usuários com DV. Dentre a pesquisa, destaca-se a documentação de cada um destes programas que, de uma forma generalizada, disponibilizava as teclas de atalho separadas por "categoria". Além disto, vários guias da utilização do gerenciador de e-mail foram encontrados.

A partir deste ponto, buscou-se elaborar o conteúdo textual para ser adicionado no Menu Auxiliar criado na primeira etapa da pesquisa (Cruz e Rodrigues, 2015). Para essa etapa levou-se em consideração dois aspectos: o usuário, ao utilizar o leitor, deverá apresentar a ele os recursos mais indispensáveis para que possa ser capaz de criar um documento simples; o usuário deverá acessar as documentações dos programas em si para aprender os detalhes, caso deseje conhecer algum dos recursos mais a fundo. Esses aspectos foram estabelecidos para que, o conteúdo apresentado fosse sucinto e objetivo e, tal ação evitava que o usuário sentisse cansado devido a uma saturação textual. Por fim, acredita-se que ao disponibilizar o meio de acesso à documentação permitiria ao usuário praticar cada vez mais a navegação por meio da internet ao utilizar o leitor de tela.

É importante salientar que, cada recurso introduzido ao software fora devidamente testado e para isso foi desligado qualquer recurso que estivesse relacionado ao uso do mouse e, além disso, a todo o tempo os olhos do investigador foram vendados. Essa ação permitia que o investigador fosse guiado apenas meio da narração do leitor de tela, com o objetivo de realizar testes realistas ao aproximar-se da forma como as pessoas com DV navegam pelo computador e como utilizariam as funcionalidades implementadas. Ao término dessa etapa passou-se a desenvolver e implementar a construção de novas janelas na interface gráfica, configuração dos botões de forma que pudessem ser "focalizados" por meio do teclado numérico e o preenchimento dessas janelas com os textos elaborados.

Para a elaboração de novas janelas, buscou-se utilizar os modelos guias já desenvolvido no começo do projeto (Cruz e Rodrigues, 2015). Tal escolha potencializou a manutenção do código e permitiu com que o mesmo pudesse ser mantido de forma organizada. Novas informações foram adicionadas para facilitar o entendimento do código devido ao seu caráter extenso. A estrutura final do software pode ser vislumbrada na figura 1. Esta corresponde a uma caixa de diálogo, composta por um texto descritivo (referente ao o que cada janela se propõe a apresentar) e botões para acessar as páginas adjacentes. Por exemplo, a janela principal apresenta cinco botões onde os quatro primeiros acessam outras 
janelas (Janela 1, 2, 3_a e 4) enquanto o quinto corresponde ao botão de "Sair". As demais caixas de diálogos seguem a mesma lógica, na estrutura apresentada pela figura em questão. Além disto, o código, ao final do desenvolvimento foi organizado em 3 partes, sendo apresentados e subdivididos da seguinte maneira: 1. Bibliotecas necessárias para execução do projeto; 2. Funções Básicas: a) Botões de retorno; b) Botões de avançar páginas; c) Funções auxiliares para manipulação das janelas; e d) Funções de rastreamento do teclado numérico. 1.Definição das janelas e seus conteúdos: a) Janela 4 e derivados; b) Janela 3 e derivados; c) Janela 2; d) Janela 1 e derivados; e) Janela Principal.

Figura 1: Esquematização de janelas do Software

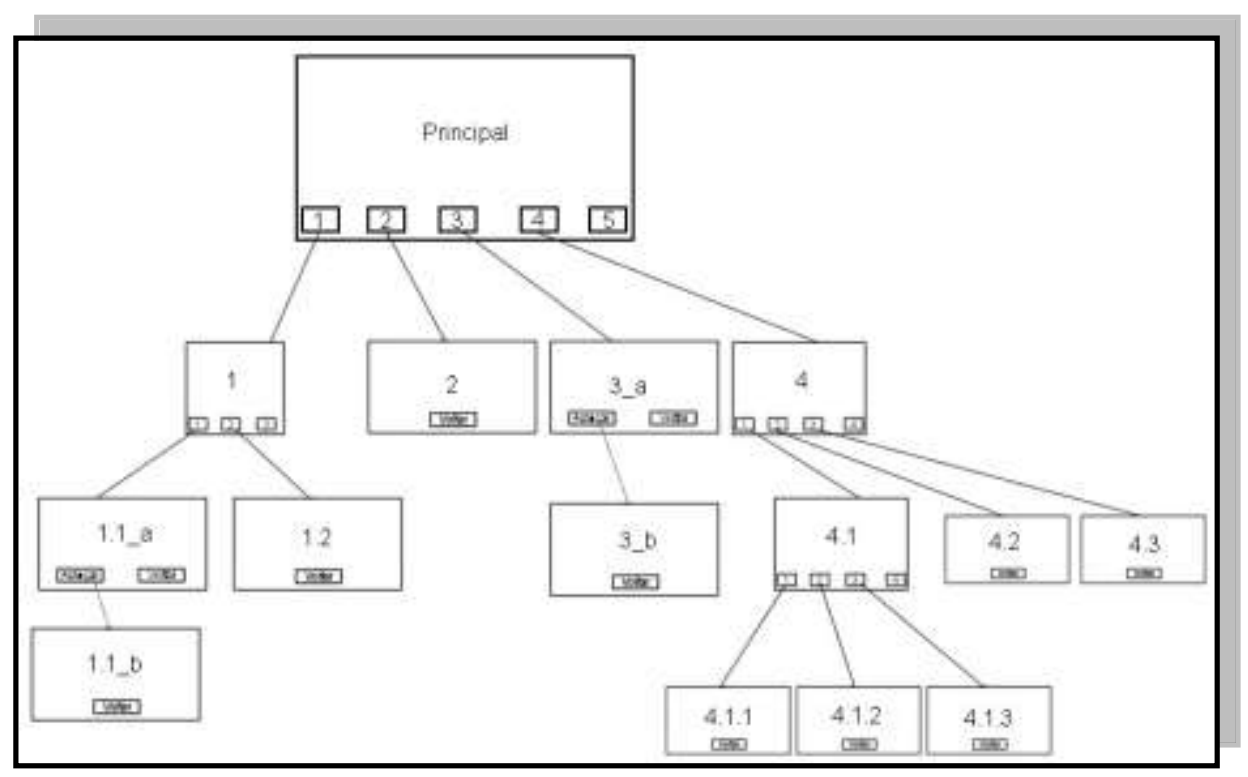

\section{RESULTADOS OBTIDOS}

Os conteúdos textuais, desenvolvidos na primeira etapa da pesquisa, foram adicionados ao software nessa segunda etapa. É importante destacar que, esse conteúdo textual possibilitava que cada um dos módulos elaborados possuíssem detalhes suficientes para permitir que o usuário com DV pudesse compreender quais seriam os recursos que uma página Web acessível disponibiliza, assim como realizar o seu primeiro acesso aos programas do pacote Office e compreender como utilizar o gerenciador de e-mails Mozilla Thunderbird (Fig. 2). 
Figura 2: Parte do conteúdo textual referente aos Recursos de Acessibilidade

\section{RECURSOS DE ACESSIBILIDADE}

Websites que possuem recursos de acessibilidade normalmente adotam algumas teclas de atalho que facilitam a navegação, seguindo sempre um mesmo padrão. Para o navegador Google Chrome, são eles:

1. Teclando-se Alt +1 em qualquer página do portal, chega-se diretamente ao começo do conteúdo principal da página

2. Teclando-se Alt +2 em qualquer página do portal, chega-se diretamente ao início do menu principal.

3. Teclando-se Alt +3 em qualquer página do portal, chega-se diretamente em sua busca interna.

Além disso, vale salientar que cada um dos módulos textuais elaborados nesse projeto possuem suas respectivas fontes que, de uma forma geral, tratam da documentação oficial dos programas e atividades explicadas. Com isto, o usuário com DV tem a oportunidade de pesquisar informações mais detalhadas nestas fontes, além de possibilitar a prática da navegação na internet e o uso das teclas de atalho.

Ao término da criação e implementação dos novos recursos ao meu auxiliar, realizouse testes com dois usuários do Centro de Apoio e Integração do Deficiente de Itajubá (CAIDI). Estes usuários apresentavam um nível conhecimento para utilizar o computador, acessar as redes sociais como, por exemplo, o facebook. Entretanto, os mesmos utilizavam o leitor de tela Jaws. Em virtude disso, não detinham pleno conhecimentos em relação as teclas de atalho do NVDA. O teste se baseou na realização de ações simples, correspondente ao que o software propunha a ensinar sendo elas: abrir o Windows Explorer, editar um documento no Microsoft Word, entre outros. Tal ação permitiu verificar a real usabilidade e acessibilidade do objeto computacional desenvolvido e, verificou-se que o menu funcionava perfeitamente. Além disso, foi realizado outro teste, mas desta vez apenas com uma pessoa com DV. Esta usuária apresenta um perfil semelhante ao dos usuários anteriores, ainda que com um grau de conhecimento muito maior por utilizar o computador diariamente. Esse teste contudo, permitiu descobrir a necessidade de alterar uma configuração do NVDA para que ele pudesse ser totalmente compatível com o Menu Auxiliar. Trata-se de uma alteração no esquema de teclado para "Portátil". Desta maneira, o teclado numérico passa a funcionar corretamente e não propicia problemas a nenhum outro comando.

Por fim, como resultado verifica-se que essa pesquisa de iniciação científica permitiu aos investigadores obter um software totalmente funcional que contém toda a informação textual construída na primeira etapa do projeto (Cruz e Rodrigues, 2015). E, que, além disso, ao usar o programa o usuário tem a possibilidade de identificar qual tecla numérica está sendo pressionada e focaliza o botão correspondente àquele número. Não obstante, todos os textos elaborados podem ser lidos com sucesso pelo leitor de tela, mediante o uso das configurações recomendadas anteriormente. O software está disponível para toda a comunidade, para isso basta acessar o site do grupo de pesquisa NEFTI, sendo ele www.nefti.com.br. 


\section{CONCLUSÕES}

Tendo em vista, a necessidade de equiparação de direitos e oportunidades, o projeto de iniciação científica desenvolvido na UNIFEI, possibilita as pessoas com DV, que estão iniciando suas atividades no mundo digital, maior autonomia para navegar na internet ou no computador quando se utiliza o NVDA. Espera-se também, que o usuário com DV possa ser capaz de explorar os recursos básicos de acesso e elaboração de documentos no Word, apresentações no PowerPoint, planilhas no Excel e acesso ao e-mail no Mozilla Thunderbird. Por meio desse projeto, conclui-se que é imprescindível desenvolver e aprimorar os recursos de Tecnologia Assistiva para que as pessoas com diferentes tipos de deficiências, síndromes e/ou transtornos possam ter acesso a diferentes setores da sociedade.

É importante salientar que, para isso se faz necessário que, os profissionais da área de Sistemas de Informação e Engenharia de Computação estejam abertos para compreender que a tecnologia pode potencializar de diferentes formas as atividades desenvolvidas pelas pessoas com Deficiência. No entanto, para que isso aconteça consideramos que, inicialmente, é preciso investir esforços em pesquisas sobre essa temática, assim como incluir na grade dos cursos de tecnologia disciplinas que abordem a temática da inclusão. Para finalizar, espera-se que essa pesquisa também desperte o interesse de novos pesquisadores e desenvolvedores para aprimorar o leitor de tela NVDA, uma vez que ele é um software aberto. 


\section{REFERÊNCIAS}

BRASIL. Constituição da República Federativa do Brasil. Brasília: Imprensa Oficial, 1988.

BRASIL. e-MAG Leitores de tela: Descrição e Comparativo. 2009

CRUZ, D.B;RODRIGUES, P.A.A. In: Sílvia Maria de Oliveira Pavão. [et al.] organizadoras. (Org.). Aprendizagem e acessibilidade: travessias do aprender na universidade. 1ed.Santa Maria: , 2015, v. 1, p. 407-416

DECLARAÇÃO DE SALAMANCA. Necessidades Educativas Especiais - NEE In: Conferência Mundial sobre NEE: Acesso em: Qualidade - UNESCO. Salamanca/Espanha: UNESCO 1994.

GALVÃO FILHO, T. A. A Tecnologia Assistiva: de que se trata? In: MACHADO, G. J. C.; SOBRAL, M. N. (Orgs.). Conexões: educação, comunicação, inclusão e interculturalidade. 1 ed. Porto Alegre: Redes Editora, p. 207-235, 2009.

OLIVEIRA JUNIOR, J. F. O. Avaliação de Acessibilidade de Softwares Leitores de Tela por Pessoas com Deficiência Visual Total com Base nas Diretrizes de Acessibilidade Para Agente de Usuário. Disponível em: <http://bsi.uniriotec.br/tcc/201304Oliveira.pdf > Acesso em: 10 Abril 2017.

MELO, R. 5 Leitores de tela para seu computador. Disponível em: <http://www.movimentolivre.org/artigo.php?id=50> Acesso em: 10 Abril 2017. 